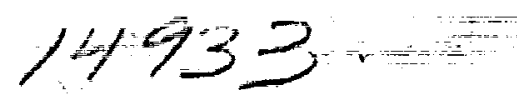

\title{
A Simplified Dynamic Model of the Space Shuttle Main Engine
}

Ahmet Duyar and Vasfi Eldem

Florida Atlantic University

Boca Raton, Florida

and

Walter Merrill and Ten-Huei Guo

Lewis Research Center

Cleveland, Ohio

Prepared for the

American Control Conference

sponsored by the American Automatic Control Council

Boston, Massachusetts, June 26-28, 1991

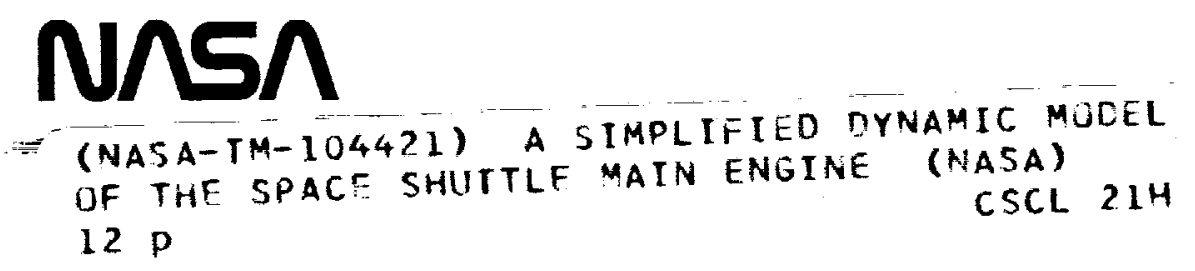

N91-23340 
..

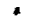




\author{
Ahmet Duyar and Vasfi Eldem \\ Florida Atlantic University \\ Department of Mechanical Engineering \\ Boca Raton, Florida 33431 \\ and \\ Walter Merrill and Ten-Huei Guo \\ National Aeronautics and Space Administration \\ Lewis Research Center \\ Cleveland, Ohio 44135
}

\begin{abstract}
Thig paper presents a simplified model of the space shuttle main engine (SSME) dynamics valid within the range of operation of the engine. This model is obtained by linking the linearized point models obtained at 25 different operating points of the SSME. The simplified model was developed for use with a model-based diagnostic scheme for failure detection and diagnostics studies, as well as control design purposes.
\end{abstract}

\title{
INTRODUCTION
}

This paper presents a simplified mathematical model describing the dynamics of the space shuttle main engine (SSME). A complete nonlinear dynamic simulation of the SSME was developed by Rockwell International Corporation (ref. 1). Due to its size and complexity, however, this nonlinear simulation is difficult to use for various design and diagnostic purposes. Therefore, a simplified dynamic model of the ssme, valid within the range of engine operation, is required for model based diagnostic and control system design.

The description of the SSME and its control system is presented in a work by Duyar, Guo, and Merrill (ref. 2). Also in a previous study, Duyar, Eldem, Merrill, and Guo (ref. 3), developed an identification algorithm and used it to obtain linearized models of the SSME at five different operating points. There the five different operating points were obtained over a range of main combustion chamber pressures at a constant value of mixture ratio. This study is a continuation which increases the number of operating point models to 25 and links perturbation models identified at these operating points to obtain a simplified model of the ssme. Here, the operating points not only include a range of main combustion chamber pressures but also a range of mixture ratios. The coefficient matrices of the linear models were regressed with the operating point parameters (chamber pressure and mixture ratio) to yield a simplified nonlinear model valid within the range considered. The accuracy of the simplified model was tested by designing an input signal which covers the whole range of operating points considered and then comparing the responses of the linked model and the nonlinear simulation. This approach has been successfully applied to control and diagnostic design in other propulsion systems (ref. 4).

Possible applications of this model include a model-based diagnostics scheme for failure detection purposes which compares the parameters of the simplified model with the identified parameters of the actual system on a continuous basis. The differences between the modeled and identified parameters may be analyzed to detect the presence, to isolate the source, and to determine the extent of ssME component 
fallures. Other applications for this model would include predictions of remaining component life, open loop engine dynamics studies, and closed loop control analysis with a user generated control law.

The remainder of this paper includes a brief description of the data acquired from the nonlinear dynamic simulation. This is followed by a description of the structure of the identified point models and the linked model. Finally, simulated results obtained from the linked model are compared with the results obtained from the nonlinear simulation for the same test input.

\section{DATA ACQUISITION}

In order to obtain input-output data of the SSME, a nonlinear dynamic simulation program was used. The main combustion chamber mixture ratio, $\mathrm{MR}_{\mathrm{N}^{\prime}}$ and pressure, $\mathrm{P}_{C \mathrm{~N}^{\prime}}$ and the engine face pressure, $\mathrm{p}_{\mathrm{a}}$, are the parameters which define a nominal operating condition. However, it was observed that the engine face pressure does not significantly affect the selected state of the output variables of the system. Hence, only the mixture ratio and the chamber pressure were selected as the defining variables.

To obtain the data at a particular operating point, the nonlinear simulation with closed-loop control was allowed to converge to the desired operating point. Then, the control was removed and the actuator outputs, oxidizer preburner oxidizer valve (OPOV) rotary motion and fuel preburner oxidizer valve (FPOV) rotary motion were disturbed simultaneously using two uncorrelated full length, pseudo-random binary sequences (PRBS). The maximum magnitude of each sequence was 2 percent of the steady-state value of the input. The clock-time interval chosen for each sequence was $0.04 \mathrm{sec}$. The length of the PRBS used was 127 intervals for each input which corresponds to a signal duration of $127 \times 0.04=5.08 \mathrm{sec}$. Thus, the chosen clocktime and signal duration give a sequence with a bandwidth from 0.2 to $12.5 \mathrm{~Hz}$.

\section{POINT MODELS}

Once the input/output data (of length 127) was obtained at each of the operating points, the developed identification algorithm was used to obtain the point models. First, a two-input/two-output model was considered. Here the outputs were the deviations of the chamber inlet pressure, $P_{C^{\prime}}$ and the mixture ratio, $M R$ and the inputs were the deviations of the valve actuator output rotary motion $\beta_{\mathrm{OPOV}}$ and $\beta_{\mathrm{FPOV}}$. From the operating point data the identification algorithm yielded a fourth order system in $a$-canonical form with observability indices $(2,2)$. This result encouraged further investigation to obtain a model with measurable state variables. To this end the linear dependences between several different outputs and $P_{c}$ and $M R$ were compared and finally high pressure fuel turbine, HPFT, and high pressure oxidizer turbine, HPOT, speeds, $S_{\text {HPFT }}$ and $S_{\text {HPOT' }}$ were chosen as third and fourth state variables for the dynamic model of the SSME. Then, the identification algorithm was employed to determine the parameters of this conceptual model. This model can be expressed mathematically as: 


$$
\delta x_{1}(k+1)=A_{1} \delta x_{1}(k)+B_{1} \delta u_{1}^{*}(k) \quad 1=1,2, \ldots, 25
$$

where $A_{1}$ and $B_{i}$ are $4 \times 4$ and $4 \times 2$ system matrices at operating point $i$ and $\delta \mathrm{x}_{1}(\cdot)$ is the model estimate of the state, which is also the model estimate of the output, since $\mathrm{C}=\mathrm{I}$. The perturbed input is defined as

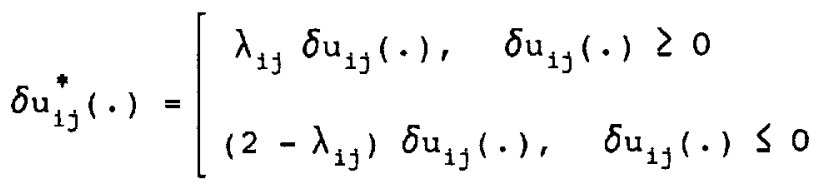

with $\delta \mathrm{u}_{11}(\cdot)$ and $\delta \mathrm{u}_{12}(\cdot)$ denoting the perturbed input values $\beta_{\mathrm{OPOV}}$ and $\beta_{\mathrm{FPov}}$ at operating point $i$, respectively. Perturbed values of the inputs are

$$
\delta u_{i j}(\cdot)=\left(u_{1 j}(\cdot)-\bar{u}_{i j}\right) / \bar{u}_{i j}
$$

where $\bar{u}_{i j}$ denotes the steady state value of input $j$ at operating point i. In a previous study (ref. 3) it was observed from the open loop response of the SSME that the gains for positive and negative perturbation signals are significantly different. In order to compensate for this phenomenon, piecewise linear system gaing, $\lambda_{1 j}$, with different values for positive and negative perturbations were added to the linearized model. These piecewise linear gains defined by equation (2) were determined by using the sample averages of outputs and inputs at each operating point as explained in reference 3 .

The perturbed values of the measured outputs of the nonlinear simulation, $\delta y_{1}(\cdot)$, at each operating point were defined as

$$
\delta y_{11}(\cdot)=\left(y_{11}-\bar{Y}_{11}\right) / \bar{Y}_{11}
$$

where

$y_{11}$ deviation of the chamber inlet pressure at operating point $i$

$\bar{y}_{11} \quad$ steady state value of the chamber inlet pressure at operating point i

Similar definitions are valid for $\delta_{y_{1 j}}(\cdot)$ where the index $j=2,3,4$ denote $M R, S_{H P F T}$ and $S_{H P O T}$ respectively.

The parameter matrices $A_{1}$ and $B_{1}$ were also employed to obtain a state filter estimate $\delta z_{1}(\cdot)$ of $\delta y_{1}(\cdot)$ using the following equation:

$$
\delta z_{1}(k+1)=A_{1} \delta Y_{1}(k)+B_{1} \delta u_{1}^{*}(k) \quad i=1, \ldots, 25
$$

THE LINKED MODEL

The coefficients of the 25 operating point models were regressed with the steady state values of $P_{C N}$ and $M R_{N}$. The variables $P_{C N}$ and ${ }^{M R_{N}}$ define an operating 
point. A second order (with respect to $P_{C N}$ and $M R_{N}$ ) polynomial expression for each coefficient was obtained. These 25 operating points correspond to five $\mathrm{MR}_{\mathrm{N}}$ levels and five power levels at each $\mathrm{MR}_{\mathrm{N}}$ level as follows:

$1-5$ correspond to $\mathrm{MR}_{\mathrm{N}}=6.026$ and $\mathrm{P}_{\mathrm{CN}}=70,80,90,100$, and 105 percent

6-10 correspond to $\mathrm{MR}_{\mathrm{N}}=6.026 \times 1.04$ and $\mathrm{P}_{\mathrm{CN}}=70,80,90,100$, and 105 percent

11-15 correspond to $M R_{N}=6.026 \times 0.96$ and $P_{C N}=70,80,90,100$, and 105 percent

$16-20$ correspond to $M_{N}=6.026 \times 1.08$ and $P_{C N}=70,80,90,100$, and 105 percent

21-25 correspond to $\mathrm{MR}_{\mathrm{N}}=6.026 \times 0.92$ and $\mathrm{P}_{\mathrm{CN}}=70,80,90,100$, and 105 percent

In regressing the coefficients however, the steady state values of the inputs and outputs at each operating point should be taken into account. This can be understood by substituting the perturbation expression of equations (2) and (3) into equation (1). Note that $\delta x_{1}(k)$ is the deviation of the model estimate of the state vector at the $i^{\text {th }}$ operating point, normalized with respect to the steady state values at that operating point defined by component as

$$
\delta x_{i j}(k)=\frac{x_{j}(k)-\bar{x}_{1 j}}{\bar{x}_{1 j}}
$$

where the subscript $j$ denotes the $j^{\text {th }}$ component of the state vector and $\bar{x}_{1 j}$ is the steady state value of the $j^{\text {th }}$ component of the state at the $i^{\text {th }}$ operating condition. Thus equation (1) can be rewritten as

$$
E_{1}^{-1}\left[x(k+1)-\bar{x}_{1}\right]=A_{1} E_{1}^{-1}\left[x(k)-\bar{x}_{1}\right]+B_{1} \Gamma_{1}^{-1}\left[u(k)-\bar{u}_{1}\right]^{*}
$$

where $\bar{x}_{i}$ and $\bar{u}_{i}$ are the steady state values of the outputs and inputs of the $i^{\text {th }}$ operating point. Here, $\bar{x}_{1}$ denotes the estimate of $\bar{y}_{1} . \quad E_{1}$ and $\Gamma_{1}$ are defined as

$$
\begin{aligned}
& \mathbf{E}_{1}=\operatorname{diag}\left\{\bar{x}_{1 j}\right\} \\
& \Gamma_{1}=\operatorname{diag}\left\{\bar{u}_{1 j}\right\}
\end{aligned}
$$

Further rearrangement of equation (7) gives

$$
x(k+1)=E_{1} A_{1} E_{1}^{-1} x(k)+E_{1} B_{1} \Gamma_{1}^{-1}\left[u(k)-\bar{u}_{1}(k)\right]^{*}+\left(I-E_{1} A_{1} E_{1}^{-1}\right) \bar{x}_{1}
$$

Thus, the coefficients to be regressed for the linked model are:

$$
E_{1} A_{1} E_{1}^{-1}, \quad E_{1} B_{1} \Gamma_{1}^{-1}, \quad \lambda_{1 j}, \bar{u}_{1} \text {, and } \bar{x}_{1} \text {. }
$$

The regregsion yields the linked model as 


$$
x(k+1)=A[x(k)] x(k)+B[x(k)]\{u(k)-\bar{u}[x(k)]\}^{*}+\{I-A[x(k)]\} \bar{x}[x(k)]
$$

for the model, and similarly

$$
z(k+1)=A[y(k)] Y(k)+B[y(k)]\{u(k)-\bar{u}[y(k)]\}^{*}+\{I-A[y(k)]\} \bar{y}[x(k)]
$$

for the linked filter. In the above equations

$$
\left[u_{j}(k)-\bar{u}_{j}(\cdot)\right]^{*}=\left[\begin{array}{c}
\lambda_{j}(\cdot)\left[u_{j}(k)-\bar{u}_{j}(\cdot)\right], \quad u_{j}(k) \geq \bar{u}_{j}(\cdot) \\
{\left[2-\lambda_{j}(\cdot)\right]\left[u_{j}(k)-\bar{u}_{j}(\cdot)\right], \quad u_{j}(k) \leq \bar{u}_{j}(\cdot)}
\end{array}\right.
$$

where (.) denotes a function of $x(k)$ for the model and $y(k)$ for the filter. Furthermore,

$$
A[\cdot]=A_{C}+A_{P} P_{C}+A_{M} M R+A_{p 2} P_{C}^{2}+A_{M 2} M R^{2}+A_{P M} P_{C} M R
$$

where $P$ and $M R$ are the current ( $k^{\text {th }}$ value) of the main combustion chamber inlet pressure and the mixture ratio. For the model the estimated values at the previous stage were used, i.e., $A[]=.A[x(k)]$. For the filter the actual outputs from the nonlinear simulation were used, i.e., $A[\cdot]=A[Y(k)]$. Note that $A_{C}$ denotes a constant term. Similar expressions can be written for $B, \lambda_{j}, \bar{u}$, and $\bar{x}$ which $y i e l d:$

$$
\begin{gathered}
B_{C^{\prime}} B_{P^{\prime}} B_{M^{\prime}} B_{P 2^{\prime}} B_{M 2^{\prime}} B_{P M^{\prime}} \lambda_{j C^{\prime}} \lambda_{J F^{\prime}} \lambda_{J M^{\prime}} \lambda_{j P 2}, \lambda_{j M 2}, \lambda_{P M^{\prime}} \\
\bar{u}_{C^{\prime}} \bar{u}_{P}, \bar{u}_{M}, \bar{u}_{P 2}, \bar{u}_{M 2}, \bar{u}_{P M^{\prime}}, \bar{x}_{C}, \bar{x}_{P^{\prime}}, \bar{x}_{M}, \bar{x}_{P 2}, \bar{x}_{M 2}, \bar{x}_{P M^{\prime}} \text { for } j=1,2 \text { (for two inputs) }
\end{gathered}
$$

The above coefficients were obtained by regressing the coefficients of the linear models with $P_{C N}$ and $M_{N}$ values of each operating point and are presented in table 1 .

The regression was performed using the statistical Package for social sciences (SPSS) (ref. 5). The chosen second degree polynomial relationship is shown in equation (14). The objective here was to include only those independent variables that were useful in predicting the dependent variable. The partial F test criterion was used to determine the contribution of an independent variable. The F statistic was calculated by testing the null and alternative hypothesis as follows:

$\mathrm{H}_{\mathrm{o}}$ : There is no relationship between the dependent variable and the independent variable under test (the coefficient under test is 0 ).

$\mathrm{H}_{1}$ : There is a definite relationship between the dependent and the independent variable (the coefficient under test is not 0 ).

Using a 95 percent level of significance, the null hypothesis is rejected if $F>F_{\text {critical }}$. The stepwise regression procedure in sPss is used to perform such a 
search. Note that the equations which define the linked model and the filter have linear structure but the coefficients are quadratic functions of $\mathrm{MR}_{\mathrm{N}}$ and $\mathbf{P}_{\mathrm{CN}}$.

\section{MODEL VERIFICATION}

The linked model was tested for accuracy by comparing its response to a test input signal with the response obtained from the nonlinear simulation.

The input test signal was designed such that it covers the whole range of operating points considered. For this purpose, input test signals for $P_{C R}$ and $M_{R}$ requests were designed first. These signals consist of steps and ramps ranging from 70 to 100 percent power levels and mixture ratio levels of 5.54 to 6.51 . Figure 1 (a) shows the $M_{R}$ and $P_{C R}$ request signals and figure $1(b)$ shows the corresponding $\beta_{\mathrm{OFOV}}$ and $\beta_{\mathrm{FPOV}}$ actuator commands generated by the controller. (Note that the same ranges for ${ }_{C R}$ and ${ }_{C R}$ were used in identifying the point models.) Then the OPOV and FPOV values corresponding to this closed-loop operation shown in figure $1(b)$ are used as inputs to the linked model (for the filter, $P_{C}$ and $M R$ values are also used). For a visual comparison, the outputs of the nonlinear simulation, $y(k)$, the model $x(k)$ and the filter, $z(k)$, are shown in figures 2 and 3 . Standard error of estimates (SEE) for both the model and the filter were also calculated according to the formula

$$
\operatorname{SEE}=\left[\frac{\Sigma[y(k)-x(k)]^{2}}{\Sigma[y(k)]^{2}}\right]^{1 / 2}
$$

Table II gives the SEE's of the predictions of both the linked model and the filter. It was observed that both the model and the filter have a good fit to the data from the nonlinear simulation.

\section{CONCLUSION}

An off-line algorithm presented in a previous study (ref. 3 ) and the data generated from the nonlinear performance simulation (ref. 1) were used to obtain linearized models of the SSME at 25 different operating points. The models have measurable state variables which make them convenient for control design and failure detection and diagnostic studies, since the need for state estimation is eliminated.

These point models were linked to obtain a simplified model of the SSME, valid within its full range of operation. The coefficients of the linear models were regressed with the parameters determining the nominal operating conditions: the chamber mixture ratio, $\mathrm{MR}_{\mathrm{N}}$, and pressure, $\mathrm{P}_{\mathrm{CN}}$. The engine face pressure, $\mathrm{p}_{\mathrm{a}}$, was also considered but it was observed not to have a significant effect on the selected state and the output variables of the system. The regression yields a nonlinear model valid within the range of mixture ratio and power levels considered. This simplified model was tested by designing an input signal which covers the whole range of operating points considered and then comparing the responses of the linked model and the nonlinear simulation. The comparison indicated good agreement between these responses. It is concluded that the simplified model is sufficiently accurate to be used in model-based diagnostics. 


\section{ACKNOWLEDGMENT}

The authors thank $J$. Litt for his review comments and the referees for their comments and suggestions which helped to improve the manuscript.

\section{REFERENCES}

1. Rockwell International Corporation, 1981, "Engine Balance and Dynamic Model," Report FSCM No. 02602, Spec. No. RL00001.

2. Duyar, A., Guo, T., and Merrill, W.C., "Space Shuttle Main Engine Model Identification," IEEE Control systems Magazine, pp. 59-65, Vol. 10, No. 4, June 1990 .

3. Duyar, A., Eldem, V., Merrill, W.C., and Guo, T., "State Space Representation of the Open Loop Dynamics of the Space Shuttle Main Engine; A Parameterization Using $a$-Canonical Form," submitted to ASME Journal of Dynamic Systems Measurement and Control, Dec. 1989.

4. Merrill, W.C., HYTESS II - A Hypothetical Turbofan Engine Simplified Simulation with Multivariable Control and sensor Analytical Redundancy. NASA TM-87344, June 1986.

5. Nie, N.H., Hadlaihul, C., Jenkins, J.G., Steinbrenner, K., and Bent, D.H., statistical Package for the Social Sciences: Manual," McGraw-Hill, 1975.

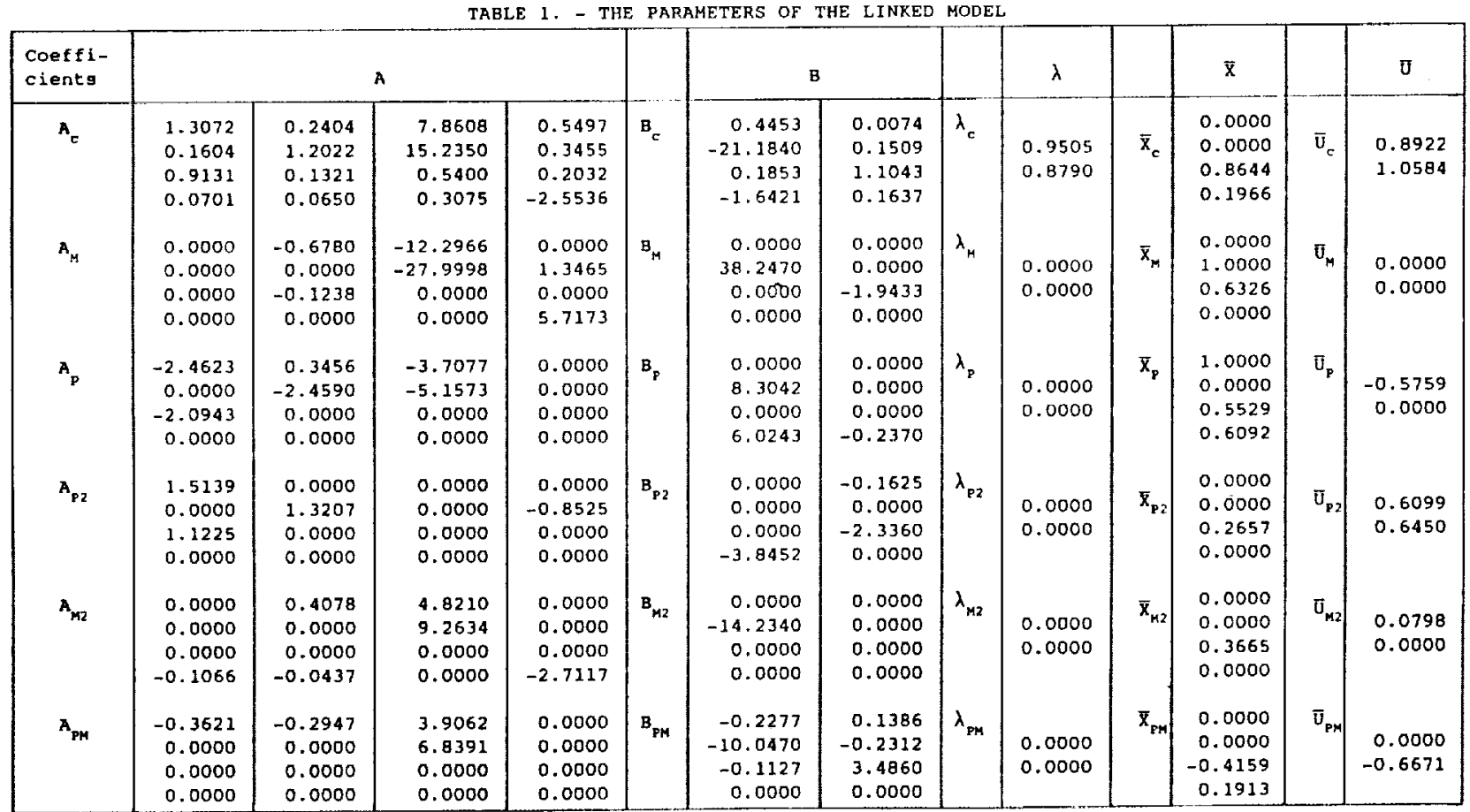


TABLE 2. - STANDARD ERROR OF ESTIMATES OF

PREDICTIONS OF THE FILTER AND THE MODEL

FOR THE TEST SIGNAL COVERING ALL

OPERATING POINTS

\begin{tabular}{|l|c|c|c|c|}
\hline \multicolumn{6}{|c|}{ Test signal } \\
\hline Output & $P_{c}$ & MR & $S_{\text {HPFT }}$ & $S_{\text {HPOT }}$ \\
\hline $\begin{array}{l}\text { Filter } \\
\text { Model }\end{array}$ & $\begin{array}{r}0.0039 \\
.0128\end{array}$ & $\begin{array}{r}0.0114 \\
.0244\end{array}$ & $\begin{array}{r}0.0076 \\
.0151\end{array}$ & $\begin{array}{r}0.0028 \\
.0075\end{array}$ \\
\hline
\end{tabular}

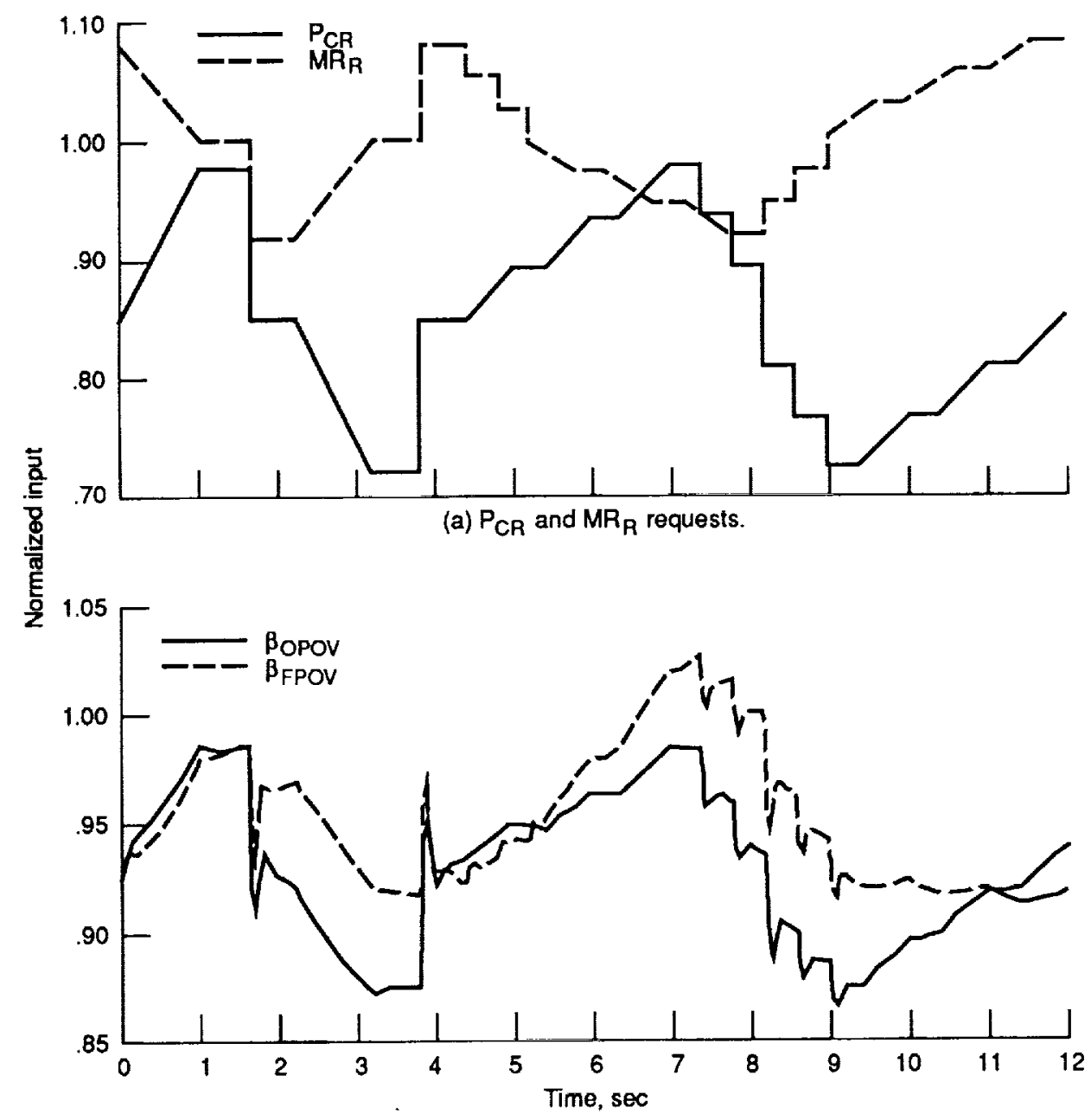

(b) $\beta_{\text {OPOV }}$ and $\beta_{\text {FPOV signals. }}$

Figure 1.- Reference input signals and their corresponding valve inputs 


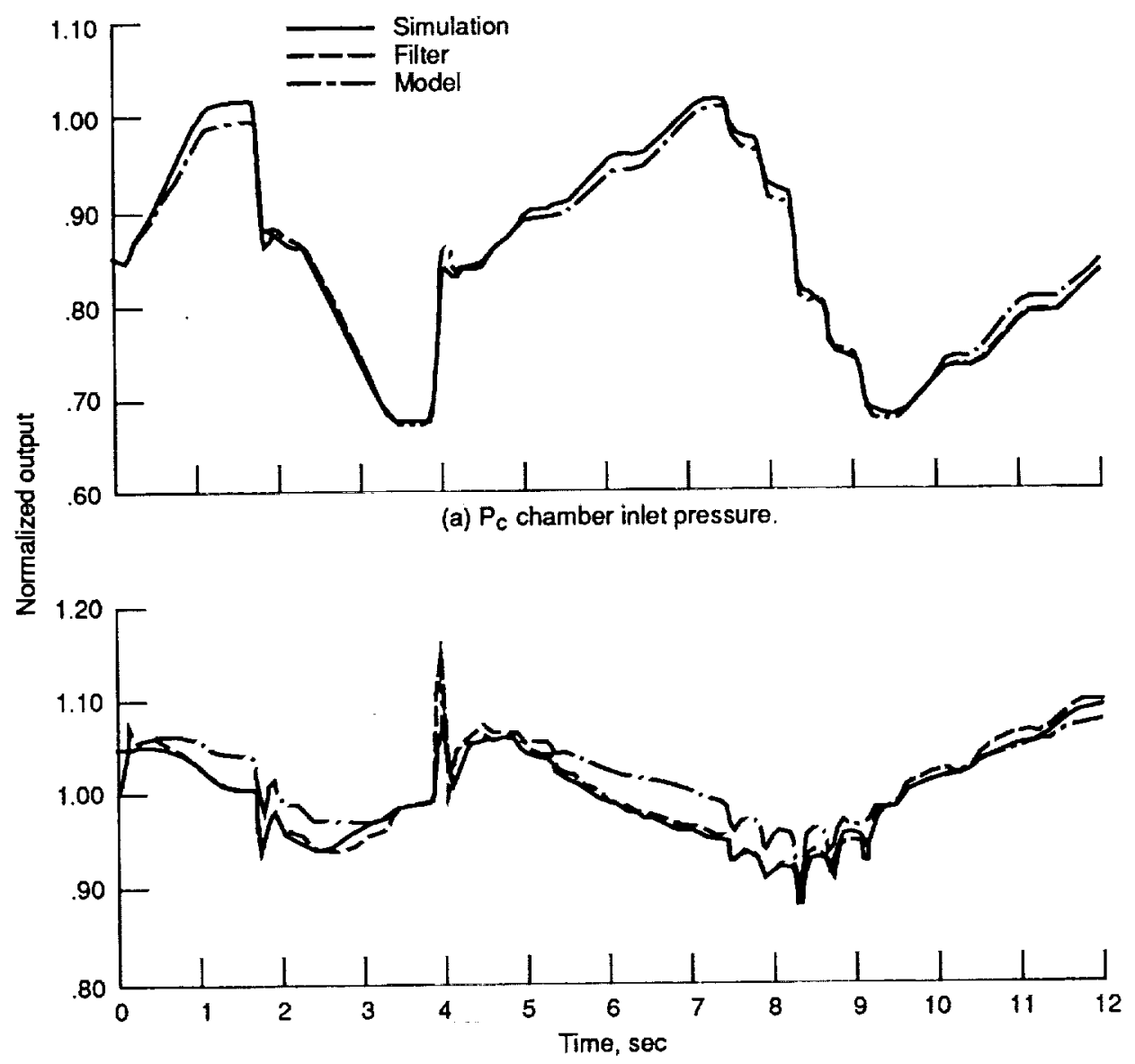

(b) MR mixture ratio.

Figure 2.-Comparison of the responses of the linked model and the filter with the nonlinear simulation. 


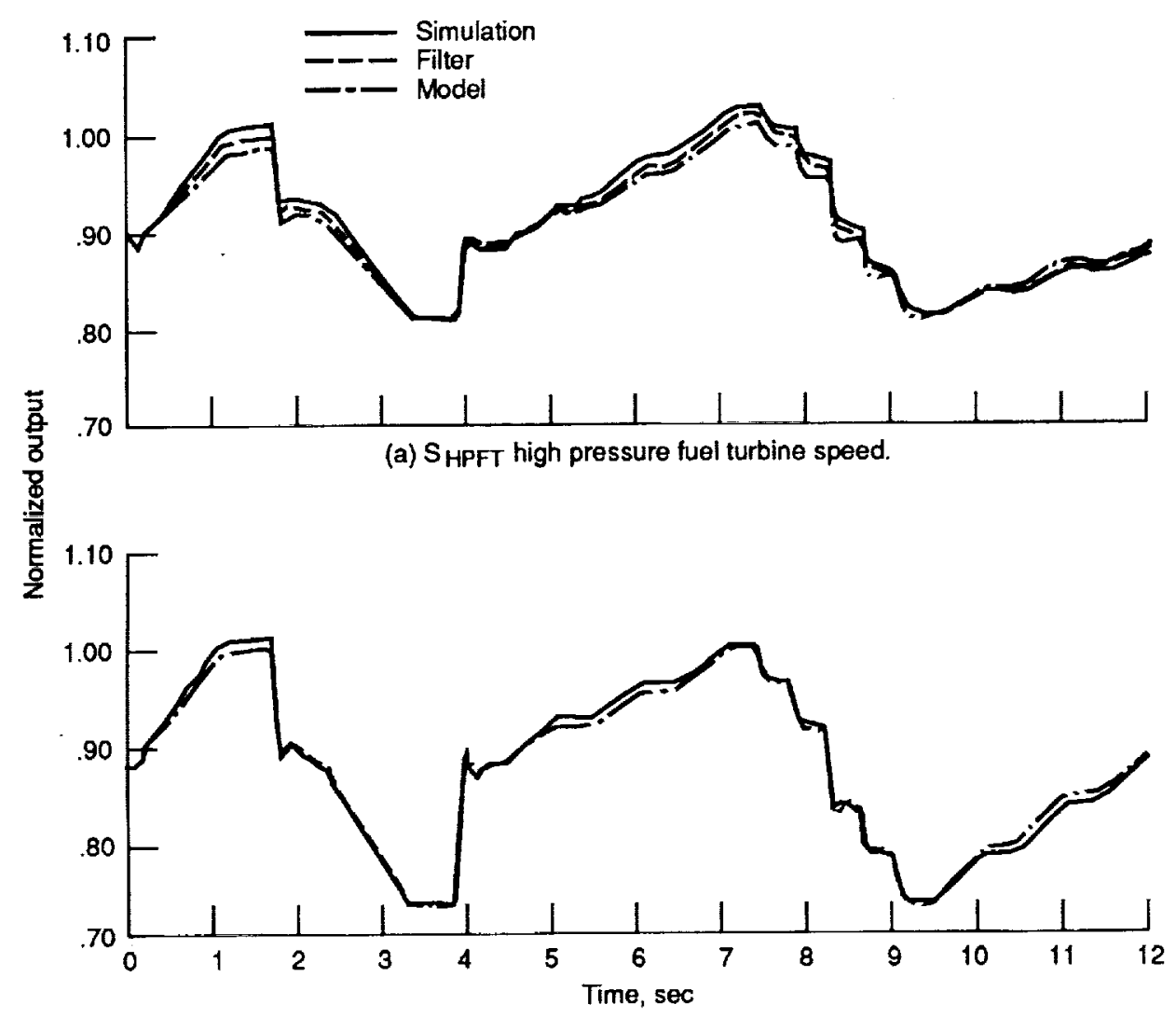

(b) SHPOT high pressure oxidizer turbine speed.

Figure 3.-Comparison of the responses of the linked model and the filter with the nonlinear simulation. 


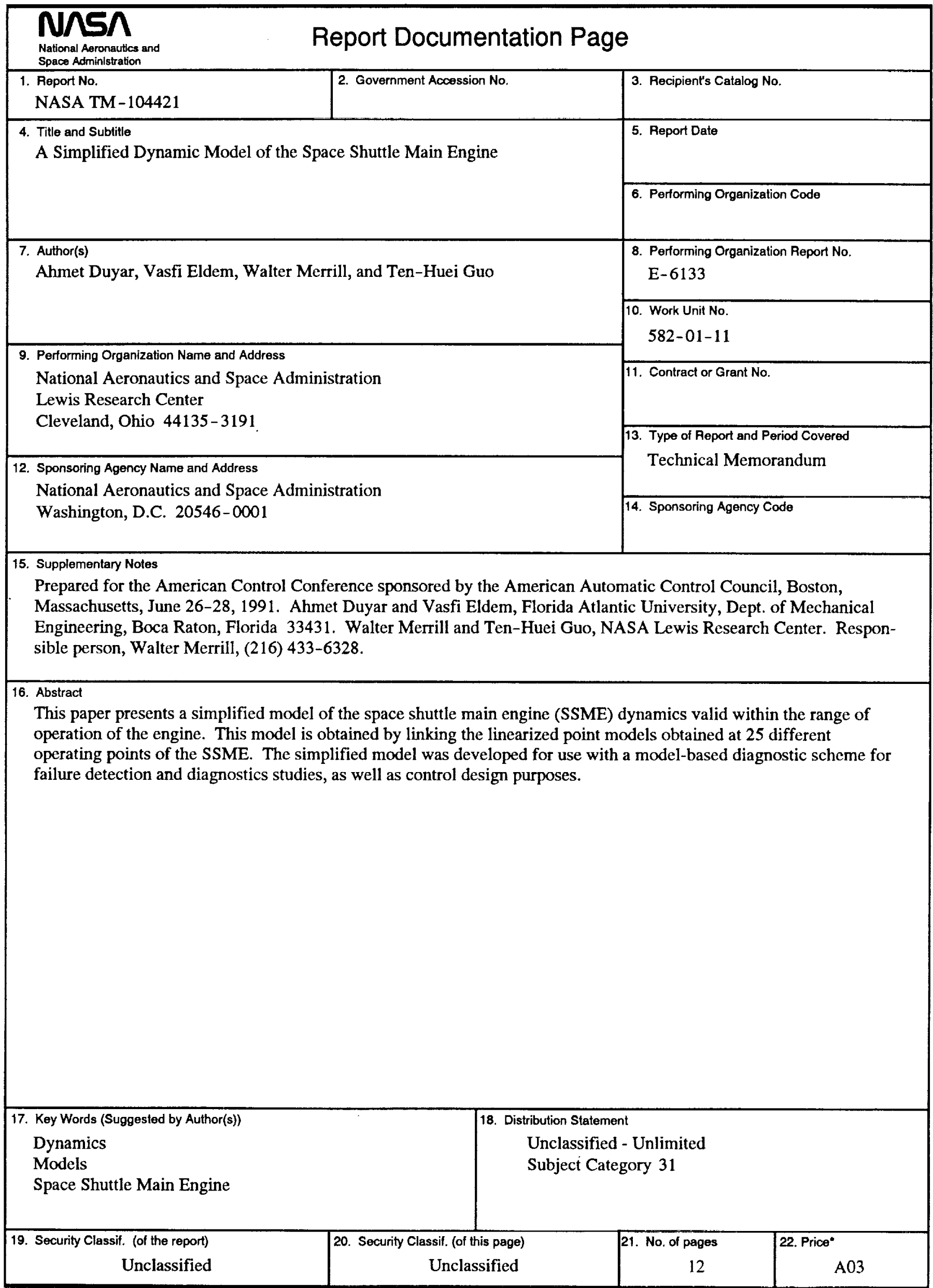


